\title{
Thermal Performance of Heat Exchanger Using Zeolite/vapour Adsorption
}

\author{
Soichiro OHNO ${ }^{*}$, Shuji HIRONAKA ${ }^{1}$, and Jun FUKAI ${ }^{1}$ \\ ${ }^{1}$ Department of Chemical Engineering, Graduate School of Engineering, Kyushu University, Ito Campus West 4 building, 744 \\ Motooka Nishi-ku Fukuoka 819-0395, Japan
}

\begin{abstract}
About $60 \%$ of the energy input in the chemical industry is discarded from the plant. Energy saving can be achieved in the entire plant by recovering these waste heats and reusing them as power and heat sources in the power plant. An adsorption heat pump has been developed for the purpose of regeneration of such unused energy. In this study, saturated humid air was supplied to a device packed with $13 \mathrm{X}$ zeolite particles of $4 \mathrm{~mm}$ in diameter. The time variation of temperature in the apparatus was measured experimentally. Then, the maximum temperature was estimated from the relationship between heat balance and adsorption equilibrium. The trend of the maximum temperature calculated from the heat balance is consistent with experiment. Further, it was found from the result of the heat balance equation that the sensible heat of the humid air supplied and the heat of adsorption of the zeolite are mainly distributed to the sensible heat of the zeolite. In the future, it is important to make effective use of the sensible heat of this zeolite. In order to extract more thermal energy from the device, it is necessary to improve the heat transfer between the packed bed and medium. A double pipe heat exchanger having a zeolite packed bed on the annular side was proposed as an apparatus. Flow direction of the humid air supplied to device was changed in two different ways. The one of them is supplying humid air radial flowly to the device and another is supplying the air in parallel flow. The influence of flow direction on heat transfer between packed bed and medium is studied with numerical simulation.
\end{abstract}

\section{Introduction}

The use of waste heat is an increasingly urgent task for modern industries such as the chemical, steelmaking and metallurgical sectors around the world. Industrial waste heat recycling not only reduces energy costs, but also reduces the release of heat to the environment. Highgrade waste heat recycling is relatively easily achieved by waste heat boilers (Zhang et al., 2013) and heat pipes (Srimuang and Amatachaya, 2012) and is already used in industry. Low-grade waste heat can be reused in Organic Rankin Cycle (Campana et al., 2013), absorption heat pumps (Horuz and Kurt, 2010), and adsorption heat pumps (Hirata et al., 2011) (AHP). In particular, large amounts of wastewater $\left(\leq 80^{\circ} \mathrm{C}\right)$ and waste gases $\left(\leq 140^{\circ} \mathrm{C}\right)$ may be found in the chemical industry (International Energy Agency, 2007).

In our previous work, the concept of direct contact heat exchange between porous adsorbents and heat exchange fluids was introduced to generate useful vapor directly from hot water (Atakan et al., 2013). This new steam generation system from zeolite-water AHP has been experimentally proven and analyzed numerically (Oktariani et al., 2012). However, there are problems with the durability of the adsorbent particles in the process of water adsorption to zeolite. One of the causes is that the water in the particle pore is boiled and the volume jumps to cause breakage of the particle. The other problem is that the adsorbent particles are deteriorated due to the binder used in forming the particles containing water. Investigations should be made to overcome the problem of durability of the adsorbent and also to improve the heat transfer coefficient of a particle packed bed with generally low effective thermal conductivity.

In this study, saturated humid air was used as the material to contact the adsorbent. This makes it possible to greatly ameliorate the aforementioned problems with particle durability. Since the thermal characteristics of the zeolite when humid air was used as the working fluid have not been studied, first, the temperature response when saturated air was allowed to flow through the zeolite particle packed bed was investigated by experiments. Secondly, the maximum temperature was estimated using heat balance equations prepared under multiple assumptions, and the dominant heat quantity from the beginning of the experiment to the maximum temperature was calculated. Finally, in a double-tube AHP generally used as a chemical heat pump, the influence of the flow direction of the moist air supplied

\footnotetext{
* Corresponding author: s.ohno-k@kyudai.jp
} 
to the particle packed bed on the heat transfer to the heat medium was investigated. Then the possibilities for heat transfer enhancement of the device were discussed. This examination was conducted by numerical simulation using numerical analysis software Fluent.

\section{Experimental}

\subsection{Set up and procedure}

Figure 1 shows a schematic of the experimental apparatus. A cylindrical reactor with a tube length of $0.35 \mathrm{~m}$ was packed with $13 \mathrm{X}$ zeolite particles with a diameter of $4 \mathrm{~mm}$. Humidification was performed by passing dry air branched from the facility utility through a bubbling tank to generate saturated humid air. This humid air was supplied to the reactor, and the temperature behaviour in the reactor due to the heat of adsorption generated was measured. The bubbling tank and the route to the reactor inlet are heated by a ribbon heater to control the temperature of the humid air. The experimental conditions are shown in Table 1.

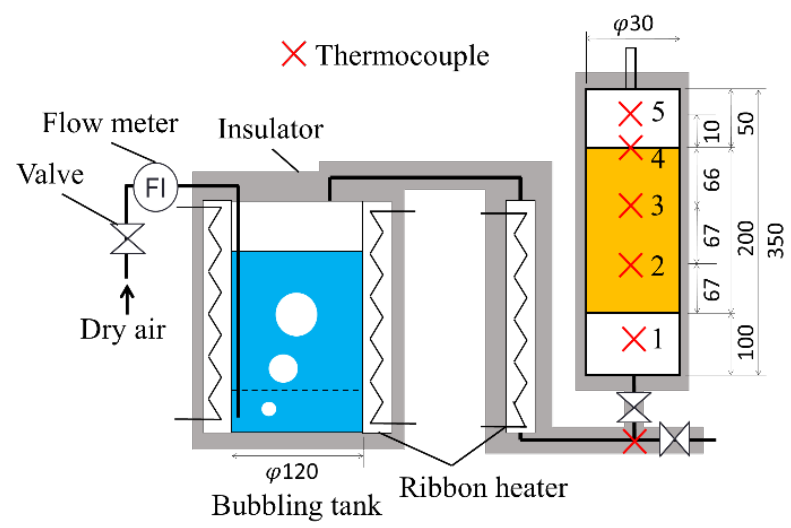

Figure 1. Schematic illustration of experimental setup

Table 1. Experimental conditions

\begin{tabular}{|l|c|c|}
\hline \multicolumn{1}{|c|}{ Parameter } & Unit & Value \\
\hline Inflowing humid air temperature & {$\left[{ }^{\circ} \mathrm{C}\right]$} & $30,50,70,90$ \\
\hline Standard superficial velocity & {$\left[\mathrm{m}_{\mathrm{N}} / \mathrm{s}\right]$} & $0.11,0.22$ \\
\hline Initial adsorption ratio & {$[\mathrm{kg}-\mathrm{w} / \mathrm{kg}-\mathrm{p}]$} & About 0.07 \\
\hline Packed bed height & {$[\mathrm{m}]$} & 0.2 \\
\hline Mass of adsorbent & {$[\mathrm{kg}]$} & About 0.11 \\
\hline Experiment time & {$[\mathrm{s}]$} & $3600 \sim 7000$ \\
\hline
\end{tabular}

\subsection{Estimation of maximum temperature}

In the period from the initial stage to the maximum temperature in the packed bed in the experimental device, the heat balance equation was created as shown in equation (1), and the calculation of the maximum temperature and the heat quantity distributed to each term were examined. The following assumptions are made for the preparation of the heat balance equation. The data obtained by experiment was used for the calculation.

- The reactor walls are insulated and there is no heat loss.
- The temperature in the packed bed rises from the initial stage to the control temperature only by the sensible heat of the humid air.

- The temperature in the packed bed rises from the control temperature to the maximum temperature only by the heat generated by adsorption.

- Specific heat does not depend on temperature and is always constant.

$$
\begin{aligned}
C_{\mathrm{H}} F & \left(T_{1}-T_{0}\right) \Delta t_{0 \sim 1}+M_{\mathrm{p}}\left(x-x_{0}\right) \Delta H_{\mathrm{z}} \\
= & M_{\mathrm{p}}\left(C_{\mathrm{p}}+C_{\mathrm{w}} x_{0}\right)\left(T-T_{0}\right) \\
& +C_{\mathrm{H}} F\left\{\left(T_{\mathrm{av} .1 \sim 2}-T_{1}\right) \Delta t_{1 \sim 2}+\left(T_{\mathrm{av} .0 \sim 1}-T_{0}\right) \Delta t_{0 \sim 1}\right\} \\
& +C_{\mathrm{r}} M_{\mathrm{r}}\left(T_{\mathrm{r} 2}-T_{0}\right)
\end{aligned}
$$

From the above assumptions and the heat balance equation, the functions for the adsorption rate $x$ and the temperature $T$ were created to calculate the maximum temperature. Figure 2 shows how to calculate the maximum temperature.

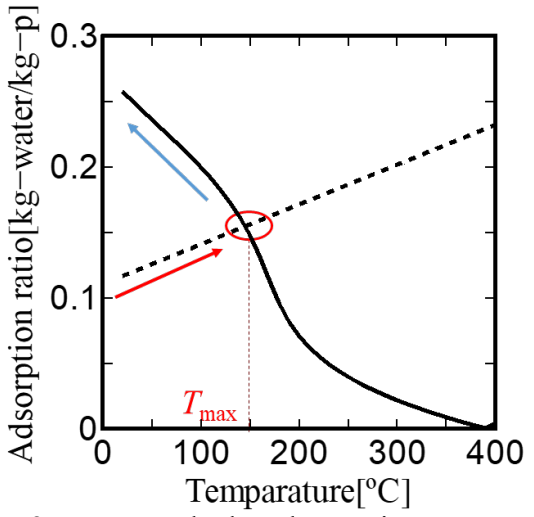

Figure 2. How to calculate the maximum temperature

First, the adsorption rate and temperature rise according to the heat balance equation (red arrow in Figure 2). Next, it intersects with the adsorption isobaric line, after which the adsorption ratio rises along the isobar and the temperature falls (blue arrow in Figure 2). Therefore, the maximum temperature can be calculated by reading the temperature at the intersection of the two graphs.

\section{Theoretical}

\subsection{Models}

Chemical heat pumps are generally systems that indirectly transfer the heat generated by the reaction to the heat medium via a heat exchanger. The equipment targeted in this research is a system that transfers the heat supplied to the equipment and the heat of adsorption generated in the zeolite particle packed bed to the heat medium. Here, in the particle-packed bed, the particles are in point contact with each other, and the void between the particles is large, which generally has a disadvantage that the effective thermal conductivity is low. In order to improve the performance of the device without increasing the device volume, it is necessary to develop a heat transfer enhancement method between the particle packed bed and the heat transfer medium. 
In this research, heat transfer acceleration method was examined by numerical simulation for double-pipe heat exchangers with simple shapes.

The main point of this numerical analysis is to change the direction of the humid air supplied to the zeolite particle packed bed of the annular part. Figure 3 shows simulation models. In a double-pipe heat exchange type heat storage device, a model is proposed that flows perpendicularly to the flow direction of the fluid in the inner pipe (Figure 3 (b)). This model can expand the heat transfer area when exchanging heat with the inner pipe compared to other models, and can expect heat transfer promotion. The heat transfer to the inner tube was investigated for these three models.

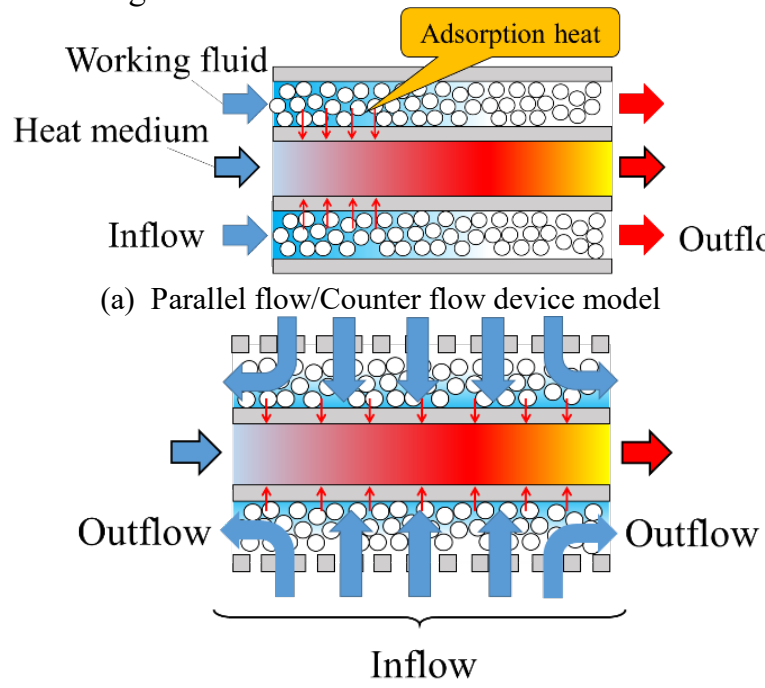

(b) Radial flow device model (Spray from around)

Figure 3. Schematic of simulation models

\subsection{Procedure}

Numerical analysis software Fluent was used for the calculation. Geometric modelling, meshing, setup, analysis execution, and post processing were performed to obtain the results. Two dimensional finite volume method was used for the analysis method.

\subsection{Computational Domain}

Figure 4 shows the calculation area in the axisymmetric coordinate system ( $\mathrm{R}-\mathrm{Z}$ axis). The inner tube side is made of water, the inner tube wall is made of stainless steel, and the shape composed of three zones of porous region modelling zeolite particle packed bed of annular part is created. Also, the annular side is the dual region of the supplied humid air and the packed bed of zeolite particles, in each of which the governing equations are solved.

\subsection{Governing Equations}

Calculations were performed under laminar flow conditions assuming an incompressible fluid. The governing equation is shown below.

Continuity

$\nabla \cdot \mathbf{v}_{\mathrm{f}}=0$

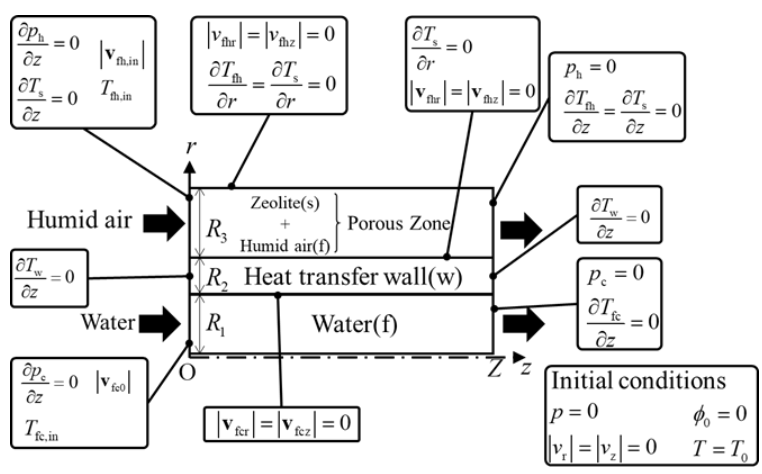

(a) Parallel/Counter flow model

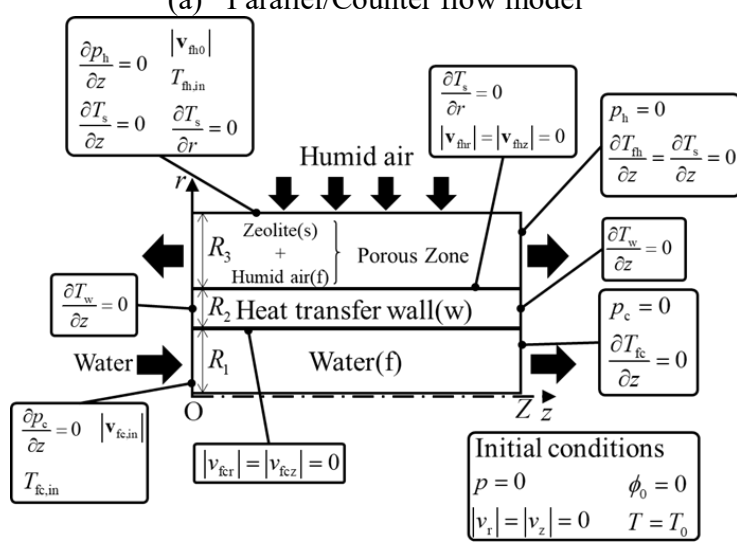

(b) Radial flow model

Figure 4. Computational domain with boundary conditions

Momentum

Annular side :

$$
\rho_{\mathrm{f}} \frac{\partial \mathbf{v}_{\mathrm{fh}}}{\partial t}+\rho_{\mathrm{f}} \nabla \cdot\left(\mathbf{v}_{\mathrm{fh}} \mathbf{v}_{\mathrm{fh}}\right)=-\nabla p_{\mathrm{h}}-\left(\frac{\mu_{\mathrm{f}}}{K} \mathbf{v}_{\mathrm{fh}}+\frac{C_{2}}{2} \rho_{\mathrm{f}}\left|\mathbf{v}_{\mathrm{fh}}\right| \mathbf{v}_{\mathrm{fh}}\right)
$$

Inner side :

$$
\rho_{\mathrm{f}} \frac{\partial \mathbf{v}_{\mathrm{fc}}}{\partial t}+\rho_{\mathrm{f}} \nabla \cdot\left(\mathbf{v}_{\mathrm{fc}} \mathbf{v}_{\mathrm{fc}}\right)=-\nabla p_{\mathrm{c}}+\mu_{\mathrm{f}} \nabla \cdot\left(\nabla \mathbf{v}_{\mathrm{fc}}+\nabla \mathbf{v}_{\mathrm{fc}}^{\mathrm{T}}\right)
$$

Energy

Annular side:

Humid air:

$$
\rho_{\mathrm{f}} C_{\mathrm{pf}} \varepsilon\left(\frac{\partial T_{\mathrm{fh}}}{\partial t}+\mathbf{v}_{\mathrm{fh}} \cdot \nabla T_{\mathrm{fh}}\right)=\varepsilon k_{\mathrm{f}} \nabla^{2} T_{\mathrm{fh}}+\frac{6(1-\varepsilon)}{d_{\mathrm{p}}} h_{\mathrm{fs}}\left(T_{\mathrm{s}}-T_{\mathrm{fh}}\right)(5)
$$

Zeolite :

$\rho_{\mathrm{s}} C_{\mathrm{ps}}(1-\varepsilon) \frac{\partial T_{\mathrm{s}}}{\partial t}=(1-\varepsilon) k_{\mathrm{s}} \nabla^{2} T_{\mathrm{s}}+\frac{6(1-\varepsilon)}{d_{\mathrm{p}}} h_{\mathrm{fs}}\left(T_{\mathrm{fh}}-T_{\mathrm{s}}\right)$

Inner side :

$$
\rho_{\mathrm{f}} C_{\mathrm{pf}} \frac{\partial T_{\mathrm{fc}}}{\partial t}+\rho_{\mathrm{f}} C_{\mathrm{pf}} \mathbf{v}_{\mathrm{fc}} \cdot \nabla T_{\mathrm{fc}}=k_{\mathrm{f}} \nabla^{2} T_{\mathrm{fc}}
$$

Inner tube :

$$
\rho_{\mathrm{w}} C_{\mathrm{pw}} \frac{\partial T_{\mathrm{w}}}{\partial t}=k_{\mathrm{w}} \nabla^{2} T_{\mathrm{w}}
$$

Here, the specific heat of the stainless steel tube used for the inner pipe and the reactor wall was given as a constant value of $590 \mathrm{~J} /(\mathrm{kg} \cdot \mathrm{K})$ and the thermal conductivity of $16.7 \mathrm{~W} /(\mathrm{m} \cdot \mathrm{K})$.

Also, the heat transfer coefficient between the adsorbent particles and the fluid is based on Wakao's equation.

$$
h_{\mathrm{fs}}=\frac{k_{\mathrm{f}}}{d_{\mathrm{p}}}\left\{2+1.1\left(\frac{\rho_{\mathrm{f}} d_{\mathrm{p}} v}{\mu}\right)^{0.6}\left(\frac{C_{\mathrm{p}} \mu}{k_{\mathrm{f}}}\right)^{1 / 3}\right\}
$$




\subsection{Simulation Conditions}

Figure 4 shows the initial conditions and boundary conditions used for the simulation. Also analysis conditions are shown in Table 2.

Table 2. Analysis conditions

\begin{tabular}{|c|c|c|}
\hline Parameter & Unit & Value \\
\hline$R_{1}$ & {$[\mathrm{~m}]$} & 0.02 \\
\hline$R_{2}$ & {$[\mathrm{~m}]$} & 0.005 \\
\hline$R_{3}$ & {$[\mathrm{~m}]$} & 0.015 \\
\hline$\varepsilon$ & {$\left[\mathrm{m}^{3}-\right.$ fluid $\left./ \mathrm{m}^{3}-\mathrm{CV}\right]$} & 0.4 \\
\hline$\phi_{\mathrm{f}}$ & {$[\mathrm{kg}$-vapor $/ \mathrm{kg}$-humidair $]$} & 0.75 \\
\hline$T_{\mathrm{fh}, \text { in }}$ & {$\left[{ }^{\circ} \mathrm{C}\right]$} & 200 \\
\hline$T_{\mathrm{s} 0}$ & {$\left[{ }^{\circ} \mathrm{C}\right]$} & 200 \\
\hline$T_{\mathrm{fc}, \text { in }}$ & {$\left[{ }^{\circ} \mathrm{C}\right]$} & 25 \\
\hline$\left|\mathbf{v}_{\mathrm{fc}, \text { in }}\right|$ & {$[\mathrm{m} / \mathrm{s}]$} & 0.025 \\
\hline$\left|\mathbf{v}_{\mathrm{fh}, \text { in }}\right|$ & {$[\mathrm{m} / \mathrm{s}]$} & 0.86 \\
\hline Parallel or Counter flow) & & \\
\hline
\end{tabular}

Here, the volumetric flow rate of the humid air supplied to the annular side is kept constant in all models (Parallel flow, Counter flow and Radial flow). In the radial flow model, the inlet flow velocity decreases as the device length increases. The relationship between the device length and the inlet flow rate is shown in Table 3. Figure 5 shows the grid shape and each device length.

Table 3. Device length and inlet flow rate (radial flow)

\begin{tabular}{|c|c|c|c|c|c|}
\hline \multirow{2}{*}{ Parameter } & \multirow{2}{*}{ Unit } & \multicolumn{4}{|c|}{ Value } \\
\cline { 3 - 6 } & & (a) & (b) & (c) & (d) \\
\hline$Z$ & {$[\mathrm{~m}]$} & 0.0122 & 0.0244 & 0.0488 & 0.122 \\
\hline$\left|\mathbf{v}_{\mathrm{fh}, \mathrm{in}}\right|($ Vertical $)$ & {$[\mathrm{m} / \mathrm{s}]$} & 0.86 & 0.43 & 0.215 & 0.086 \\
\hline & \multicolumn{1}{l}{} \\
\hline
\end{tabular}

(b)

(c)

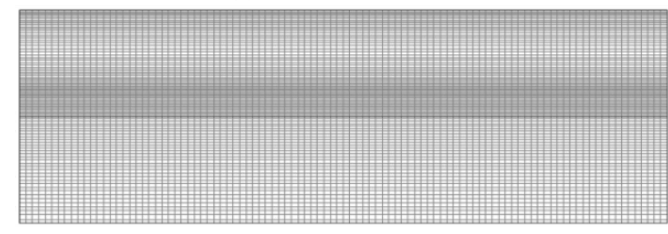

(d)

Figure 5. Grid shape and device length

\section{Results and Discussion}

\subsection{Experimental}

\subsubsection{Effect of temperature of humid air}

Figure 6 shows the influence of the inlet humid air temperature on the time-dependent change of the temperature inside the packed bed. The numbers in Figure 4 indicate the numbers of thermocouples in
Figure 1. When the control temperature of the humid air rises, the maximum temperature becomes higher due to the sensible heat of the inlet humid air.

In addition, the maximum temperature is reached faster at the beginning of the experiment. This is because the amount of water vapor in the supplied humid air increases, and the adsorption rate increases.

The ability to raise the temperature in the packed bed to around 150 to $200{ }^{\circ} \mathrm{C}$ was confirmed by using saturated humid air.
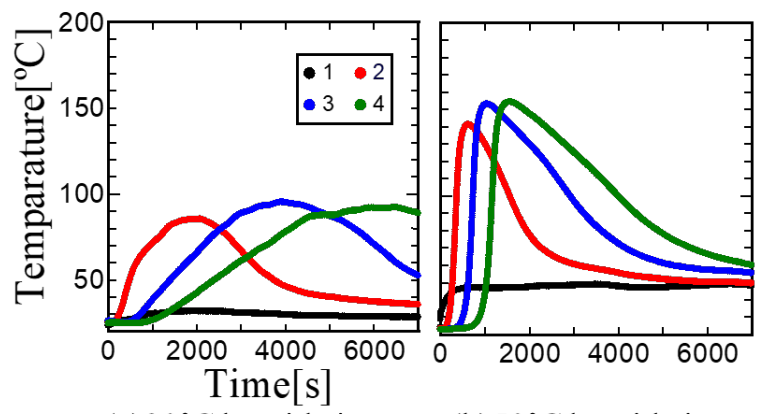

(a) $30^{\circ} \mathrm{C}$ humid air

(b) $50^{\circ} \mathrm{C}$ humid air
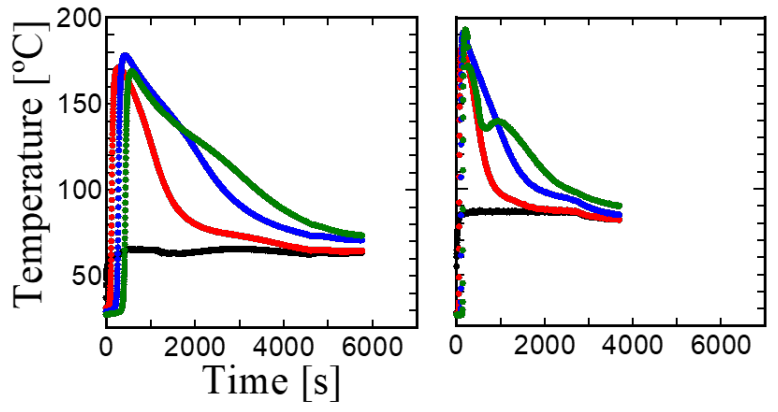

(c) $70^{\circ} \mathrm{C}$ humid air

(d) $90^{\circ} \mathrm{C}$ humid air

Figure 6. Influence of inlet humid air temperature $\left(0.11 \mathrm{~m}_{\mathrm{N}} / \mathrm{s}\right)$

\subsubsection{Effect of humid air velocity}

Figure 7 shows the influence of the flow velocity on the time-dependent change of the temperature inside the packed bed at a humid air temperature of $70{ }^{\circ} \mathrm{C}$. When the flow rate is increased, the maximum temperature is reached faster in the early stage of the experiment. This is because the adsorption rate increases because the amount of water passing through in unit time increases with the increase in flow velocity. In addition, after reaching the maximum temperature, the amount of heat carried away by air also increases, so the speed of settling to the control temperature also increases.

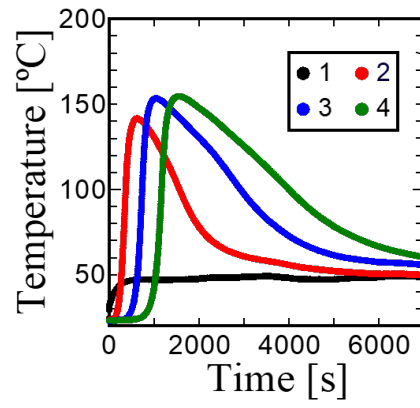

(a) $0.11 \mathrm{~m}_{\mathrm{N}} / \mathrm{s}$

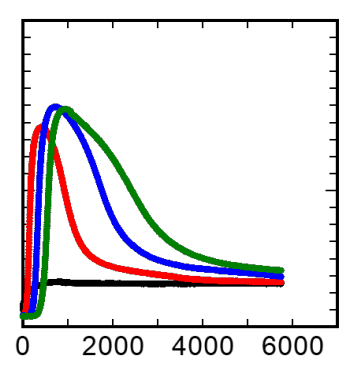

(b) $0.22 \mathrm{~m}_{\mathrm{N}} / \mathrm{s}$
Figure 7. Influence by flow velocity $\left(70^{\circ} \mathrm{C}\right.$ saturated humid air) 


\subsubsection{Estimation of maximum temperature}

The comparison between the calculated maximum temperature and the experimental value is shown in Table 4. For each flow velocity and control temperature, values close to the experimental results were obtained with an error of less than $11 \%$. Thus, the equation and assumptions used to calculate the heat balance in the period from the beginning of the experiment to the maximum temperature are generally valid.

(a) $0.11 \mathrm{~m}_{\mathrm{N}} / \mathrm{s}$

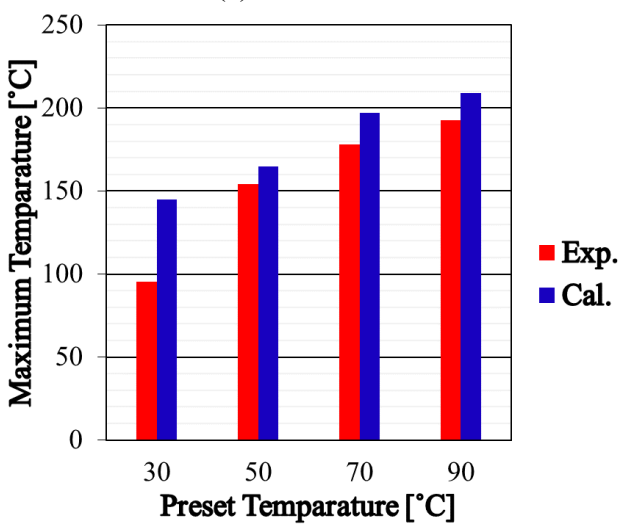

(b) $0.22 \mathrm{mN} / \mathrm{s}$

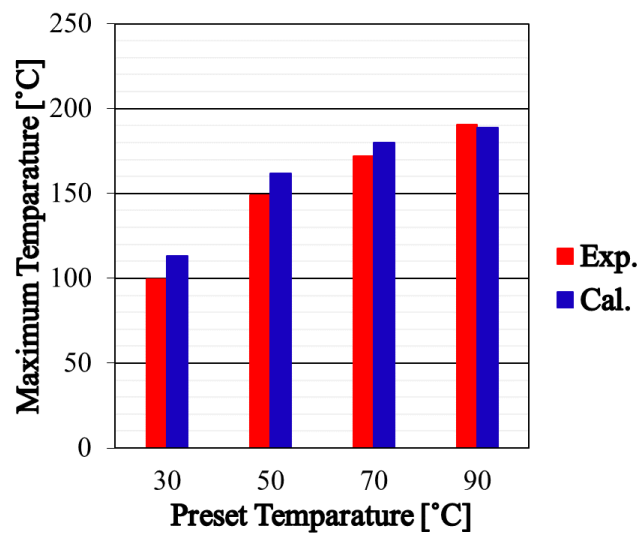

Figure 8. Comparison between experimentand calculation of maximum temperature

The heat distribution of each term in the heat balance equation with a flow velocity of $0.11 \mathrm{~m}_{\mathrm{N}} / \mathrm{s}$ is shown in Table 5. Among the distributed heat, the sensible heat of the zeolite accounts for the majority. The same tendency was also obtained at a flow velocity of $0.22 \mathrm{~m}_{\mathrm{N}} / \mathrm{s}$. By efficiently extracting the amount of heat due to this sensible heat, it leads to the development of a higher performance adsorption heat pump.

Table 4. Amount of heat distributed to each section $(0.11$ $\mathrm{mN} / \mathrm{s}$ )

\begin{tabular}{|l|c|c|c|}
\hline & $\mathbf{5 0}^{\circ} \mathbf{C}$ & $\mathbf{7 0}^{\circ} \mathrm{C}$ & $\mathbf{9 0}^{\circ} \mathbf{C}$ \\
\hline Zeolite sensible heat $[\mathrm{kJ}]$ & 16.92 & 20.11 & 21.94 \\
\hline Carry out sensible heat by air [kJ] & 8.67 & 2.01 & 3.64 \\
\hline Reactor sensible heat $[\mathrm{kJ}]$ & 13.66 & 9.93 & 13.27 \\
\hline
\end{tabular}

\subsection{Simulation}

In software, the time step size was set to 0.01 and the number of time steps was set to 30000 and calculations were performed for $300 \mathrm{~s}$. All conditions became steady at $t=300 \mathrm{~s}$. The bulk temperature at the outlet of the inner tube at $t=300 \mathrm{~s}$ and the average pressure drop at the annulus were output. The bulk temperature of the inner pipe outlet at each device length are shown in Table 6.

Table 5. Inner tube outlet bulk temperature

\begin{tabular}{|c|c|c|c|c|c|}
\hline & $\mathrm{Z}$ & 0.0122 & 0.0244 & 0.0488 & 0.122 \\
\hline Parallel & \multirow{3}{*}{$T_{\mathrm{b}}\left[{ }^{\circ} \mathrm{C}\right]$} & 25.077 & 25.137 & 25.195 & 25.312 \\
\cline { 4 - 6 } & & 25.096 & 25.138 & 25.198 & 25.317 \\
\cline { 4 - 6 } Counter & 25.038 & 25.088 & 25.137 & 25.223 \\
\hline Radial & & & &
\end{tabular}

The bulk temperature increases as the device length increases. This is the effect of the expansion of the heat transfer area. Moreover, it became high in order of counter flow, parallel flow, and radial flow in all conditions. This is because as the length of the apparatus increases, the effect of decreasing the wet air inlet flow rate increases in the radial flow model. Figure 8 shows a method of calculating the average pressure loss. The average pressure drop of the annulus at each device length is shown in Table 7.

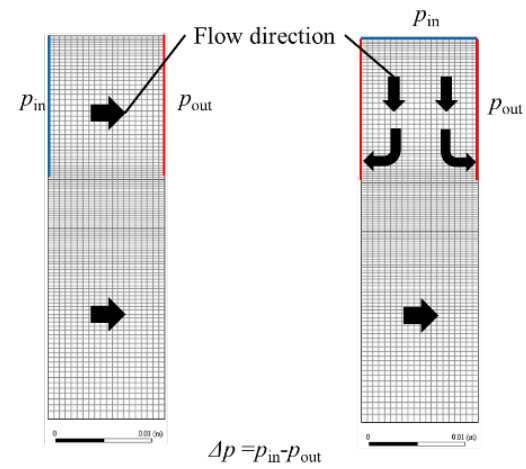

Figure 9. Calculation method of average pressure drop

Table 6. The average pressure drop of the annulus

\begin{tabular}{|c|c|c|c|c|c|}
\hline & $\mathrm{Z}$ & 0.0122 & 0.0244 & 0.0488 & 0.122 \\
\hline Parallel & \multirow{3}{*}{$\Delta p[\mathrm{~Pa}]$} & 28.616 & 56.843 & 112.928 & 279.204 \\
\cline { 4 - 7 } & 28.612 & 56.844 & 112.938 & 279.207 \\
\cline { 4 - 7 } Counter & 7.712 & 4.462 & 7.124 & 14.29 \\
\hline Radial & & &
\end{tabular}

The pressure drop was the lowest value in the radial flow model for all conditions. From this result, the newly proposed model (radial flow) can reduce the pressure loss much more than the counter or parallel flow model with the same device shape.

\section{Conclusion}

In this study, the temperature behaviour when the zeolite was brought into contact with moist air was shown by experiments, and the heat balance confirmed an effective amount of heat when removed from the apparatus. Then, for the purpose of extracting a larger amount of heat from the device, a study was conducted focusing on the flow direction of the moist air which is the working fluid. The results are summarized as follows. 
- Basic data on the thermal behaviour of contacting $13 \mathrm{X}$ zeolite with moist air was obtained.

- It is possible to estimate the amount of heat distributed from the beginning of the experiment to the maximum temperature by several assumptions and heat balance equations.

- In the double-pipe type AHP, when the inlet volumetric flow rate of humid air is unified, the heat transfer performance is the lowest in the Radial flow model.

- The average pressure drop shows a very small value for the Radial flow model (up to $93.7 \%$ can be reduced under the same equipment conditions). Therefore, if the flow velocity is determined so that the average pressure drop of the device is unified in all models, the heat transfer promotion effect by the Radial flow model can be expected.

\section{Acknowledgement}

This work was supported by JSPS KAKENHI Grant Number JP18H03856.

\section{Nomenclature}

$C_{2}=$ Coefficient of inertia resistance

$C_{\mathrm{p}}=$ Specific heat at constant pressure $[\mathrm{J} /(\mathrm{kg} \cdot \mathrm{K})]$

$d=$ Diameter [m]

$F \quad=$ Mass flowrate $\quad[\mathrm{kg} / \mathrm{s}]$

$h=$ Heat transfer coefficient $\quad\left[\mathrm{W} /\left(\mathrm{m}^{2} \cdot \mathrm{K}\right)\right]$

$K=$ Permeability $\left[\mathrm{m}^{2}\right]$

$k=$ Thermal conductivity $\quad[\mathrm{W} /(\mathrm{m} \cdot \mathrm{K})]$

$p=$ Pressure $\quad[\mathrm{Pa}]$

$R \quad=$ Radius $\quad[\mathrm{m}]$

$s \quad=$ Specific surface area $\quad[1 / \mathrm{m}]$

$T=$ Temperature $[\mathrm{K}]$

$\mathbf{v}=$ Flow velocity $[\mathrm{m} / \mathrm{s}]$

$x=$ Water content in particles $\quad[\mathrm{kg}-\mathrm{w} / \mathrm{kg}-\mathrm{p}]$

$Z=$ Length in $z$ direction $[\mathrm{m}]$

$\Delta t=$ Size of time step [s]

$\Delta H_{\mathrm{z}}=$ Adsorption heat $\quad[\mathrm{J} / \mathrm{kg}-\mathrm{w}]$

$\varepsilon \quad=$ Porosity [-]

$\phi \quad=$ Mass fraction $[-]$

$\mu=$ Viscosity $\quad[\mathrm{Pa} \cdot \mathrm{s}]$

$\rho \quad=$ Density $\quad\left[\mathrm{kg} / \mathrm{m}^{3}\right]$

Subscripts

$\mathrm{H} \quad=$ Saturated humid air

$0=$ Initial

$1=$ Time to reach control temperature

$2=$ Time to reach maximum temperature

av. = Average

$\mathrm{r} \quad=$ Reactor

$\mathrm{p} \quad=$ Particle

$\mathrm{f} \quad=$ Fluid

$\mathrm{h}=$ Hot fluid

$\mathrm{c} \quad=$ Cold fluid

$\mathrm{p} \quad=$ Particle

in $=$ Inlet

out $=$ Outlet

$\mathrm{b}=$ Bulk average

\section{References}

Atakan, A., G. Fueldner, G. Munz, S. Henninger, and M. Tatlier; "Adsorption Kinetics and Isotherms of Zeolite Coatings Directly Crystallized on Fibrous Plates for Heat Pump Applications," Appl. Therm. Eng., 58, 273-280 (2013)

Campana, F., M. Bianchi, L. Branchini, A. De Pascale, A. Peretto, M. Baresi, A. Fermi, N. Rossetti, and R. Vescovo; "ORC Waste Heat Recovery in European Energy Intensive Industries: Energy and GHG Savings," Energy Convers. Manage., 76, 244-252 (2013)

Hirata, K. and H. Kakiuchi; "Energy Saving for Ethylene Process by Adsorption Heat Pump," Appl. Therm. Eng., 31, 2115-2122 (2011)

Horuz, I. and Bener Kurt; "Absorption heat transformers and an industrial application," Renewable Energy, 35, 2175-2181 (2010)

International Energy Agency; Tracking Industrial Energy Efficiency and $\mathrm{CO}_{2}$ Emissions, pp. 279-282, Stedi Media, Paris, France (2007)

Oktariani, E., A. Noda, K. Nakashima, K. Tahara, B. Xue, K. Nakaso, and J. Fukai; "Potential of a Direct Contact Adsorption Heat Pump System for Generating Steam from Waste Water," Int. J. Energy Res., 36, 1077-1087 (2012)

Srimuang, W. and P. Amatachaya; "A Review of the Applications of Heat Pipe Heat Exchangers for Heat Recovery," Renewable Sustainable Energy Rev., 16, 4303-4315 (2012)

Zhang, H., H. Wang, X. Zhu, Y.J. Qiu, K. Li, R. Chen, and Q. Liao; "A Review of Waste Heat Recovery Technologies towards Molten Slag in Steel Industry," Appl. Energy, 112, 956-966 (2013) 